\title{
Structural phase stability and electron-phonon coupling in lithium
}

\author{
Amy Y. Liu \\ Department of Physics, Georgetown University, Washington, DC 20057-0995 \\ Andrew A. Quong \\ Lawrence Livermore National Laboratory, Livermore, California 94550 \\ J. K. Freericks \\ Department of Physics, Georgetown University, Washington, DC 20057-0995 \\ E. J. Nicol \\ Department of Physics, University of Guelph, Guelph, Ontario, Canada N1G 2W1 \\ Emily C. Jones \\ Department of Physics, Georgetown University, Washington, DC 20057-0995
}

(Received 14 September 1998)

\begin{abstract}
First-principles calculations of the free energy of several structural phases of Li are presented. The densityfunctional linear-response approach is used to calculate the volume-dependent phonon frequencies needed for computing the vibrational free energy within the quasiharmonic approximation. We show that the transformation from a close-packed structure at low temperatures to the bcc phase upon heating is driven by the large vibrational entropy associated with low-energy phonon modes in bcc Li. In addition, we find that the strength of the electron-phonon interaction in Li is strongly dependent on crystal structure. The coupling strength is significantly reduced in the low-temperature close-packed phases as compared to the bcc phase, and is consistent with the observed lack of a superconducting transition in Li. [S0163-1829(99)11905-2]
\end{abstract}

\section{INTRODUCTION}

The pressure-temperature structural phase diagram of solid Li, arguably the simplest metal, is not well established. Even along the zero-pressure axis, Li exhibits some complex behavior. At ambient pressure and room temperature, $\mathrm{Li}$ crystallizes in the bcc structure, but upon cooling, it undergoes a martensitic transformation around $80 \mathrm{~K}$. The transformation was first observed in $1956,{ }^{1}$ but the crystal structure of the low-temperature phase remained a subject of debate for several decades. In 1984, Overhauser suggested that the neutron scattering data were consistent with the 9R structure, ${ }^{2}$ a close-packed phase with a nine-layer stacking sequence. Subsequent investigations confirmed 9R as the primary structure at low temperatures, ${ }^{3}$ but also showed that it is accompanied by a large and variable amount of a disordered polytype consisting of short-ranged fcc- and hcp-type stacking order, as well as some amount of bcc. ${ }^{4}$ Furthermore, upon heating, the $9 \mathrm{R}$ phase and disordered polytype appear to transform first to an ordered fcc phase before reverting to bcc Li above about $150 \mathrm{~K} .^{4}$

Little information is available about the phase diagram of $\mathrm{Li}$ at higher pressures. Upon compression, the temperature at which the martensitic transformation begins, $M_{s}$, rises at a rate of about $20 \mathrm{~K} / \mathrm{GPa}$, at least up to pressures of $3 \mathrm{GPa}$, with the structural characteristics of the low-temperature phase remaining the same as at ambient pressure. At room temperature, a structural transition is observed at $P$ $=6.9 \mathrm{GPa}^{7}$ The crystal structure of the compressed phase was initially identified as fcc, but the limited diffraction data are also consistent with hcp or $9 \mathrm{R}$.

Neutron experiments ${ }^{3,8,9}$ show that while there may be some softening of certain phonon modes in bcc Li upon cooling, the transformation occurs well before any phonon frequencies or elastic constants approach zero, ruling out the soft-mode mechanism ${ }^{10-12}$ for the transformation. The transformation more likely results from a competition between the internal energies and entropies of the different phases. For example, Friedel ${ }^{13}$ argued on general grounds that the phonon spectrum should scale roughly with coordination number, leading to an overall lowering of the bcc phonon spectrum compared to that of close-packed structures. The resulting larger vibrational entropy associated with the bcc structure provides a qualitative explanation for the prevalence of the bcc structure as the high-temperature phase in metals. While these ideas are believed to apply to many systems that exhibit martensitic transformations, it is useful to examine them in the context of detailed materials-specific phonon spectra which are accessible from first-principles calculations.

Several first-principles theoretical investigations have focused on the relative stability of different crystal structures for Li at zero pressure. ${ }^{14,15}$ Conflicting results have emerged, but all the studies agree that the energy differences between structures are small (much less than $1 \mathrm{mRy} /$ atom), making it difficult to determine the ordering reliably. Most of the theoretical work has been based on zero-temperature calculations that do not even include zero-point energies. Given that $\mathrm{Li}$ is such a light atom, finite-temperature effects arising from vibrational degrees of freedom are likely to play a role 
in the structural energetics of this system. In a recent study, vibrational contributions to the free energies of bcc and fcc $\mathrm{Li}$ were computed within the harmonic approximation. ${ }^{15}$ The fcc phase was found to be favored at $T=0 \mathrm{~K}$, which is consistent with experiments. Furthermore, the free-energy difference between the two phases was calculated to decrease with increasing temperature, but, in contrast to what is observed in experiments, the fcc phase was calculated to remain thermodynamically stable up to at least a few hundred kelvin.

In this paper, we go beyond the purely harmonic approximation and include anharmonic effects at the quasiharmonic level. ${ }^{16}$ This approximation, in which the volume dependence of the phonon spectrum is explicitly included, has been shown to provide a remarkably good description of the temperature dependence of the lattice constant and bulk modulus of bcc Li up to its melting point. ${ }^{17}$ The densityfunctional linear response method ${ }^{18,19}$ is used to compute the volume-dependent phonon frequencies. We find that a quasiharmonic treatment of the phonon free energies reproduces the experimentally observed bcc-9R transformation, though the transition temperature is overestimated. The thermodynamic stabilization of the bcc structure at high temperatures is shown to be completely attributable to its excess phonon entropy.

A possibly related issue is the lack of a superconducting transition in Li. Specific heat measurements, ${ }^{20}$ as well as first-principles calculations ${ }^{21-25}$ suggest that the electronphonon mass enhancement parameter in $\mathrm{Li}$ is $\lambda \approx 0.45$. Using the McMillan equation, ${ }^{26}$ with a Coulomb repulsion parameter of $\mu^{*}=0.12$, which is believed to be typical for simple metals, we would expect to find a superconducting transition in $\mathrm{Li}$ at temperatures on the order of $1 \mathrm{~K}$. Yet no transition has been observed in experiments, at least down to $6 \mathrm{mK} .^{27}$

Because of uncertainties in the low-temperature crystal structure, most of the previous calculations, as well as the analysis of the specific heat data, were based on bcc Li. One calculation of the electron-phonon coupling strength in $9 \mathrm{R} \mathrm{Li}$ suggested that the coupling is indeed weaker in the lowtemperature phase. ${ }^{24}$ However, there were large uncertainties in that result since it was based on frozen-phonon calculations in which it was only feasible to examine the coupling constants at a few phonon wave vectors. Using the linearresponse approach rather than the frozen-phonon method, we are able to efficiently sample wave vectors throughout the Brillouin zone, and more accurately calculate the structure dependence of the electron-phonon interaction in $\mathrm{Li}$ in this work. Our results show that in $9 \mathrm{R}$, fcc, and hcp $\mathrm{Li}, \lambda$ is significantly smaller than in bcc Li. We argue that the calculated coupling in the close-packed phases is weak enough to be consistent with current experimental limits on the transition temperature.

The remainder of this paper is organized as follows. In Sec. II, we outline the methods used to compute the free energies and present our results for the structural phase stability of Li. Section III focuses on the comparison of the electron-phonon interaction in bcc and $9 \mathrm{R} \mathrm{Li}$. A summary and concluding remarks are given in Sec. IV.

\section{STRUCTURAL PHASE STABILITY OF LITHIUM}

\section{A. Method}

The zero-temperature structural energetics are calculated using the plane-wave pseudopotential method within the lo- cal density approximation (LDA). The Li pseudopotential is generated by the Troullier-Martins method. ${ }^{28}$ The Wigner interpolation formula is used for the correlation potential, ${ }^{29}$ and a partial core correction is used to treat the nonlinearity of the exchange and correlation interaction between the core and valence charge densities. ${ }^{30}$ Since the structures lie very close in energy, large sets of $\mathbf{k}$ points are needed to reliably compare the total energies of different structures. We use $1120,1122,1300$, and 918 points in the bcc, 9R, fcc, and hcp irreducible Brillouin zones (IBZ), respectively, to obtain total energies that are converged to within about $10^{-5} \mathrm{Ry} /$ atom with respect to $\mathrm{BZ}$ sampling. In addition, a large plane-wave cutoff of $20 \mathrm{Ry}$ is used in the calculation of total energies.

The free energy at finite temperature has contributions from both electronic excitations as well as lattice vibrations. While the effects of thermal excitation of electrons are expected to be weak at the temperatures of interest, their contributions to the free energy can easily be included by using the Mermin functional $F_{\mathrm{el}}(V, T)=E_{\mathrm{el}}-T S_{\mathrm{el}}$ rather than the standard DFT total energy. ${ }^{31}$ The lattice excitations are treated within the quasiharmonic approximation, ${ }^{16}$ where the full Hamiltonian at a given volume is approximated by a harmonic expansion about the equilibrium atomic positions, but anharmonic effects are included through the explicit volume dependence of the vibrational frequencies. The Helmholtz free energy is then given by

$$
F(V, T)=F_{\mathrm{el}}(V, T)+k_{B} T \sum_{\mathbf{q} \nu} \ln \left[2 \sinh \left(\frac{\hbar \omega_{\mathbf{q} \nu}(V)}{2 k_{B} T}\right)\right],
$$

where $\omega_{\mathbf{q} \nu}(V)$ is the frequency of the $\nu$ th phonon band at the point $\mathbf{q}$ in the Brillouin zone.

The density-functional linear-response method is used to compute the phonon spectra for each structure at several different volumes. The dynamical matrices are computed using the scheme described in Ref. 19, where the self-consistent change in the Hamiltonian caused by ionic displacements is obtained by solving a Bethe-Saltpeter equation for the change in the charge density. At each volume, the dynamical matrix is computed for $285,240,98$, and 95 phonon wave vectors in the bcc, fcc, 9R, and hcp IBZs, respectively. A planewave cutoff of $12 \mathrm{Ry}$ is used in the calculation of phonon spectra.

\section{B. Results and discussion}

The equilibrium volumes and energies calculated for bcc, fcc, hcp, and 9R Li are listed in Table I. For volumes within about $\pm 12 \%$ of the equilibrium volume, we find that the stacking of the close-packed planes in $9 \mathrm{R} \mathrm{Li}$ is near ideal: when structural parameters are chosen to correspond to ideal stacking, the calculated forces on the atoms are less than $10^{-4} \mathrm{Ry} / \mathrm{au}$ and the differences between diagonal components of the stress tensor are less than $2 \times 10^{-6} \mathrm{Ry} / \mathrm{au}^{3}$. This is consistent with neutron data which show the $c / a$ ratio to be within $0.1 \%$ of the ideal value. ${ }^{3}$ Similarly, for the hcp structure, the optimal value for $c / a$ is found to be 1.63 , which is very close to the ideal close-packed value of 1.633 .

The $T=0 \mathrm{~K}$ static calculations, which include only the LDA total energies, show bcc Li to be the least favorable of all the structures considered. Bcc Li lies about $0.1 \mathrm{mRy} /$ atom 
TABLE I. Equilibrium volume and energy calculated for bcc, fcc, 9R, and hcp Li. Results are presented for both static zerotemperature LDA calculations, as well as zero-temperature calculations that include zero-point energies. For hcp Li, only static results are presented because full quasiharmonic calculations were not performed for this phase.

\begin{tabular}{|c|c|c|c|c|}
\hline & \multicolumn{2}{|c|}{$T=0$ static } & \multicolumn{2}{|c|}{$T=0$ with $\mathrm{ZP}$} \\
\hline & $V_{0}$ & $E_{0}-E_{0}^{\mathrm{bcc}}$ & $V_{0}$ & $F_{0}-F_{0}^{\mathrm{bcc}}$ \\
\hline $\mathrm{bcc}$ & 133.2 & 0.000 & 136.3 & 0.000 \\
\hline fcc & 132.7 & -0.146 & 135.9 & -0.113 \\
\hline $9 \mathrm{R}$ & 132.8 & -0.130 & 136.1 & -0.100 \\
\hline hcp & 132.8 & -0.123 & & \\
\hline
\end{tabular}

above the close-packed structures, all three of which have nearly the same energy. We find the lowest lying structure to be fcc, with $9 \mathrm{R}$ and hcp Li only about $0.01 \mathrm{mRy} /$ atom higher in energy.

The relative ordering of various structures for Li has been the subject of some controversy. Several static LDA studies have been carried out, with varying results. ${ }^{14,15}$ Because the different calculations differ in the basis sets employed, the sampling of the BZ, the form of the LDA potential, and the shape approximations used, it is difficult to sort out the reasons for the discrepancies. Nevertheless, all the calculations agree that the structures are close in energy, and hence are at least qualitatively consistent with the coexistence of various phases at low temperatures. Given the structural similarity between the various close-packed phases, their near degeneracy in this monovalent $s p$ metal is not surprising. While the calculated total-energy differences between the closepacked phases are too small to allow us to definitively determine their relative ordering, we are confident in our result that the close-packed phases are clustered close in energy, and that all are more stable than the bcc phase.

The average phonon frequency is calculated to be about $1 \%$ lower in bcc Li than in the close-packed phases. Thus when zero-point energies are included, the energy difference between bcc Li and the close-packed phases decreases. We find however that the relative ordering of the structures remains the same. As can be seen in Table I, the inclusion of zero-point energy also leads to an expansion in the equilibrium volume. For bcc Li, the static LDA energies underestimate the volume by nearly $7 \%$. Since the atoms are so light, vibrational effects are significant, and when zero point motion is taken into account, the error in the calculated volume becomes about $4.5 \%$, which is more typical of the size of errors found in LDA studies of other simple metals.

Since the close-packed phases are virtually indistinguishable energetically, we choose the 9R structure, which is the predominant low-temperature phase, as representative of all the close-packed structures. For the remainder of this section, we focus on comparing the energetics of bcc and 9R Li.

The Helmholtz free energy for bcc and 9R Li are plotted as a function of volume in Fig. 1. Results are presented for $T=0 \quad \mathrm{~K}$ and $T=300 \mathrm{~K}$. For each crystal structure, calculations are carried out for six to eight volumes, and the results are fit to the Birch equation of state. ${ }^{32}$ With increasing temperature, the ordering of the phases reverses, and the bcc phase is calculated to be stable at room temperature, as ob-

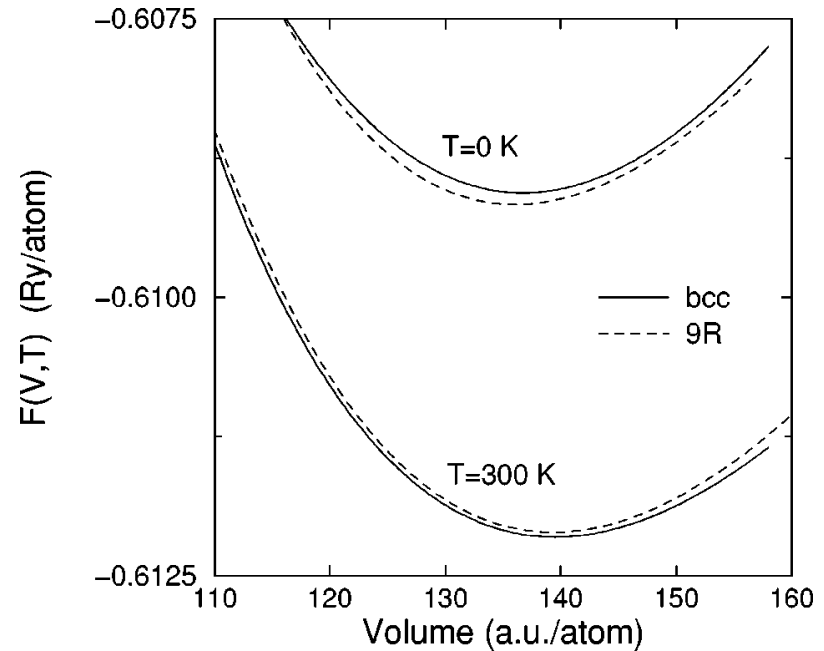

FIG. 1. Calculated Helmholtz free energy versus volume for bcc and $9 \mathrm{R} \mathrm{Li}$ at two temperatures. The curves are fits of the calculated points to the Birch equation of state.

served experimentally. Interestingly, this close-packed to bcc transition was not found in a recent LDA calculation based on the purely harmonic approximation. ${ }^{15}$ In that work, free energies for bcc and fcc $\mathrm{Li}$ were compared, and fcc $\mathrm{Li}$ was found to be energetically favored all the way from $T=0 \mathrm{~K}$ up to several hundred kelvin. In the present work, we find that the temperature range over which the close-packed phase is favored indeed expands if the volume dependence of the phonon spectrum is neglected, but we still find the bcc phase to be favored over both fcc and $9 \mathrm{R} \mathrm{Li}$ at $300 \mathrm{~K}$.

To pin down the transition temperature, it is useful to examine the Gibbs free energy at zero pressure. The difference between the 9R and bcc zero-pressure Gibbs energies, $\Delta G_{\text {tot }}(P=0, T)$, is plotted as a function of temperature in Fig. 2. The calculation indicates that $9 \mathrm{R} \mathrm{Li}$ is thermodynamically stable below about $T=200 \mathrm{~K}$. Based on the uncertainties in the calculated energy differences, we estimate error bars of about $\pm 50 \mathrm{~K}$ on the calculated transition tempera-

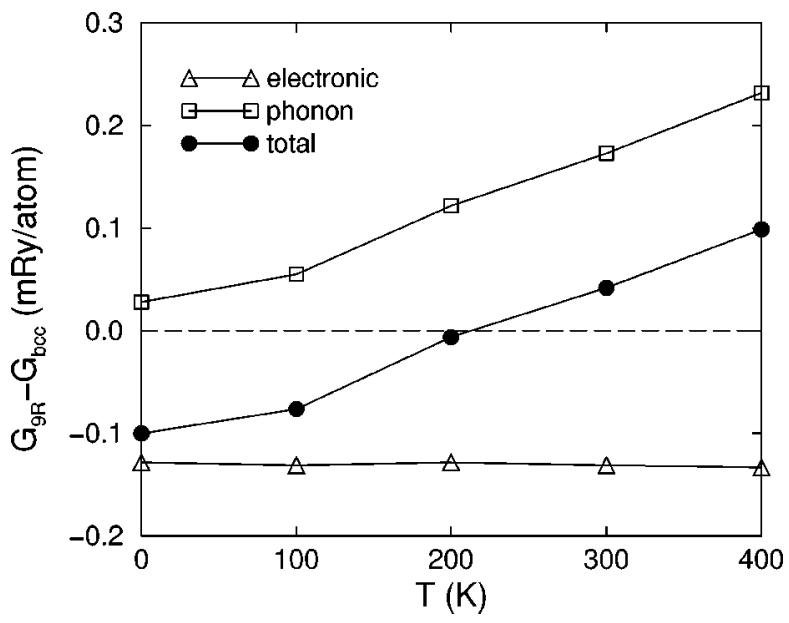

FIG. 2. Calculated difference in Gibbs free energy between 9R and bcc $\mathrm{Li}$ at zero pressure, plotted as a function of temperature. The triangles show the difference in electronic contributions to the free energy and the squares show the difference in phonon contributions. 
TABLE II. Electronic and electron-phonon-related properties computed for bcc, fcc, 9R, and hcp Li: the electronic density of states at the Fermi level $N\left(E_{F}\right)$, a logarithmic average of the phonon frequencies $\omega_{\log }$, the electron-phonon mass enhancement parameter $\lambda$, and the minimum value of the Coulomb pseudopotential, $\mu_{\min }^{*}$, that is consistent with the observed lack of a superconducting transition above $6 \mathrm{mK}$.

\begin{tabular}{lcccc}
\hline \hline & $\begin{array}{c}N\left(E_{F}\right) \\
(\text { states/Ry/spin/atom })\end{array}$ & $\begin{array}{c}\omega_{\log } \\
(\mathrm{meV})\end{array}$ & $\lambda$ & $\mu_{\text {min }}^{*}$ \\
\hline bcc & 3.33 & 14.9 & 0.45 & 0.27 \\
fcc & 3.75 & 20.2 & 0.37 & 0.21 \\
9R & 3.57 & 20.6 & 0.34 & 0.19 \\
hcp & 3.60 & 20.7 & 0.33 & 0.19 \\
\hline \hline
\end{tabular}

ture. Since the transformation actually takes place via nucleation and growth of the close-packed phase within the bcc matrix, the experimentally observed starting temperature for the transition upon cooling, about $80 \mathrm{~K}$, provides a lower bound on the thermodynamic transition temperature. Similarly, the experimental starting temperature for the closepacked to bcc transformation upon heating, about $150 \mathrm{~K}$, serves as an upper bound. ${ }^{33}$

The triangles in Fig. 2 show the 9R-bcc difference in the electronic contributions to the free energy, $\Delta G_{\mathrm{el}}(P=0, T)$ $=\Delta E_{\mathrm{el}}-T \Delta S_{\mathrm{el}}$. This quantity shows negligible temperature dependence and favors the close-packed 9R phase throughout the range of temperatures considered. In fact, $\Delta G_{\mathrm{el}}$ is dominated by the difference in internal energies, $\Delta E_{\mathrm{el}}$, which is over an order of magnitude larger than $T \Delta S_{\text {el }}$ at $T$ $=300 \mathrm{~K}$. At low temperatures, the leading term in the electronic entropy is proportional to the electronic density of states at the Fermi level $N\left(E_{F}\right)$. In this case of a simple monovalent metal, the electronic density of states depends weakly on crystal structure (Table II), and hence the electronic entropy plays a negligible role in the structural transition.

The squares in Fig. 2 show the difference between the 9R and bcc phonon free energies. The difference in vibrational energies between bcc and $9 \mathrm{R} \mathrm{Li}$ is virtually temperature independent, at least up to $T=400 \mathrm{~K}$, and it is the entropic term in the free energy that stabilizes the bcc structure with increasing temperature. The phonon densities of states, $F(\omega)$, for the two phases are plotted in Fig. 3(a). While the maximum phonon frequency is nearly the same in the two structures, the bcc phonon spectrum has more weight in the low-frequency regime (below about $15 \mathrm{meV}$ ). This enhanced weight arises primarily from the $T_{1}[\xi \xi 0]$ phonon branch, corresponding to a $[1 \overline{1} 0]$ polarization, and surrounding regions in the BZ. The entire $T_{1}[\xi \xi 0]$ branch lies below 8.5 $\mathrm{meV}$, and none of the close-packed structures have comparable low-energy phonon branches. These low-energy shear modes in bcc Li are easy to excite and give large contributions to the vibrational entropy. Over the range of temperatures considered, the vibrational entropy arising from phonon modes with frequency smaller than $8.5 \mathrm{meV}$ is about twice as large in bcc $\mathrm{Li}$ than in $9 \mathrm{R} \mathrm{Li}$, while the entropy associated with higher-frequency modes is about the same in the two structures. From the thermodynamic standpoint, the closepacked to bcc transformation in $\mathrm{Li}$ is therefore driven com-
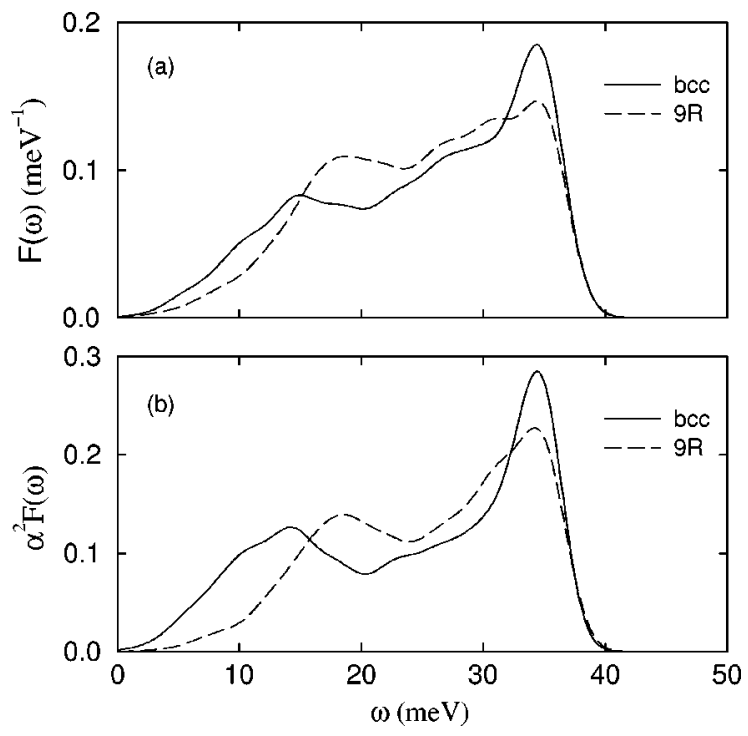

FIG. 3. (a) Phonon density of states (per atom) and (b) electronphonon spectral function calculated for bcc and $9 \mathrm{R} \mathrm{Li}$.

pletely by the excess vibrational entropy arising from the low-energy shear modes in the bcc phase.

The Clausius-Clapeyron equation allows us to estimate the slope of the phase line from the changes in entropy and volume at the transition: $d T / d P=\Delta V / \Delta S=23 \mathrm{~K} / \mathrm{GPa}$ at $P=0 \mathrm{GPa}$. This is in good agreement with the experimentally determined values for the pressure dependence of the onset temperature, which range from $d M_{s} / d T=15 \mathrm{~K} / \mathrm{GPa}$ to $30 \mathrm{~K} / \mathrm{GPa}^{5,6}$ In principle, the entire phase line can be determined by analyzing the free energies at different pressures. However, as noted in Refs. 34 and 15, the LDA predicts a negative Gruneisen parameter for the $T_{1}[\xi \xi 0]$ branch in bcc $\mathrm{Li}$, and an elastic instability at volumes corresponding to pressures of $P>2.5 \mathrm{GPa}$ at $T=0 \mathrm{~K}$ (or $P>3.1 \mathrm{GPa}$ at $T=300 \mathrm{~K}$ ). Experimentally, bcc $\mathrm{Li}$ indeed becomes unstable upon compression, and transforms to a close-packed phase, but the transformation occurs at a higher pressure of about $7 \mathrm{GPa}$. The underestimation of the transition pressure indicates that anharmonic effects beyond the quasiharmonic level become increasingly important with compression, and a treatment that includes phonon-phonon interactions that can stabilize the bcc structure is needed. ${ }^{35}$

\section{ELECTRON-PHONON COUPLING STRENGTH IN LITHIUM}

\section{A. Method}

The matrix element for scattering of an electron from state $n \mathbf{k}$ to state $n^{\prime} \mathbf{k}^{\prime}$ by a phonon with frequency $\omega_{\mathbf{q} \nu}$ and eigenvector $\hat{\epsilon}_{\mathbf{q} \nu}$ is given by

$$
g\left(n \mathbf{k}, n^{\prime} \mathbf{k}^{\prime}, \mathbf{q} \nu\right)=\sqrt{\frac{\hbar}{2 M \omega_{\mathbf{q} \nu}}}\left\langle n \mathbf{k}\left|\hat{\epsilon}_{\mathbf{q} \nu} \cdot \delta V_{\mathrm{SCF}}\right| n^{\prime} \mathbf{k}^{\prime}\right\rangle,
$$

where $\delta V_{\mathrm{SCF}}$ is the self-consistent change in the potential due to atomic displacements. This scattering gives rise to a finite phonon linewidth,

$$
\gamma_{\mathbf{q} \nu}=2 \pi \omega_{\mathbf{q} \nu}\left[N\left(E_{F}\right)\right]^{2}\left\langle\left\langle\left|g_{\mathbf{q} \nu}\right|^{2}\right\rangle\right\rangle,
$$


where the double brackets $\langle\langle\rangle\rangle$ denote a doubly constrained Fermi surface average as defined in Ref. 36. This scattering process also contributes to the effective mass of the electrons via the mass enhancement parameter

$$
\lambda_{\mathbf{q} \nu}=\frac{\gamma_{\mathbf{q} \nu}}{\hbar \pi N\left(E_{F}\right) \omega_{\mathbf{q} \nu}^{2}} .
$$

The total mass enhancement parameter, $\lambda$, is obtained by summing over branches and averaging over wave vectors throughout the Brillouin zone.

Within the Eliashberg theory of superconductivity, the electron-phonon spectral function, which measures the effectiveness of phonons of a given energy to scatter electrons on the Fermi surface, plays a central role. The spectral function is given by

$$
\alpha^{2} F(\omega)=\frac{1}{2 \pi N\left(E_{F}\right)} \sum_{\mathbf{q} \nu} \delta\left(\omega-\omega_{\mathbf{q} \nu}\right) \frac{\gamma_{\mathbf{q} \nu}}{\hbar \omega_{\mathbf{q} \nu}} .
$$

Within this framework, the mass enhancement parameter is proportional to the inverse-frequency moment of the spectral function.

In the linear-response approach, $\delta V_{\mathrm{SCF}}$ is computed in the process of determining the dynamical matrices. Therefore we calculate the matrix elements on the same grid of phonon wave vectors used in the phonon calculations described in the previous section. The doubly constrained Fermi surface average of $g$ is computing using Bloch functions at 728 (650) $\mathbf{k}$ points in the bcc (9R) IBZ, with delta functions at the Fermi level replaced by Gaussians of width $0.02 \mathrm{Ry}$. All of our studies of the electron-phonon interaction are carried out at the experimental volume for bcc $\mathrm{Li}$ at $78 \mathrm{~K}$ since previous work has shown that this yields phonon frequencies that agree well with measured dispersion curves. ${ }^{25}$ Furthermore, extrapolations based on available thermal expansion data suggest the equilibrium volume changes by less than $0.5 \%$ between $T=0 \mathrm{~K}$ and $T=78 \mathrm{~K} \cdot{ }^{37}$

The calculated $\alpha^{2} F$ functions are used as input to the Eliashberg equations. By solving the Eliashberg equations, we obtain the superconducting transition temperature $T_{c}$ as a function of the Coulomb pseudopotential $\mu^{*}$. The Eliashberg equations are solved numerically on the imaginaryfrequency axis with a cutoff of $\omega_{c}=6 \omega_{\max }$, where $\omega_{\max }$ is the maximum phonon frequency. The repulsive Coulomb parameter $\mu^{*}$ is a function of the cutoff frequency, and the value used in the numerical solution of the Eliashberg equations is not necessarily what should be used in approximate $T_{c}$ equations such as McMillan's. ${ }^{26}$ In fact, it is not completely clear which value of $\mu^{*}$ is most appropriate for such purposes, but a value scaled to $\omega_{\max }$ is usually reasonable. ${ }^{38}$ For the results reported here, $\mu^{*}$ is scaled to $\omega=\omega_{\max }$ according to $1 / \mu *\left(\omega_{\max }\right)=1 / \mu *\left(\omega_{c}\right)+\ln \left(\omega_{c} / \omega_{\max }\right)$ $=1 / \mu^{*}\left(\omega_{c}\right)+1.792$.

\section{B. Results and discussion}

The computed electron-phonon spectral functions for bcc and $9 \mathrm{R} \mathrm{Li}$ are plotted in Fig. 3(b). The fcc and hcp $\alpha^{2} F$ curves resemble the $9 \mathrm{R}$ results and are omitted for clarity. For each structure, the spectral function is similar in shape to the corresponding phonon density of states, and as with the
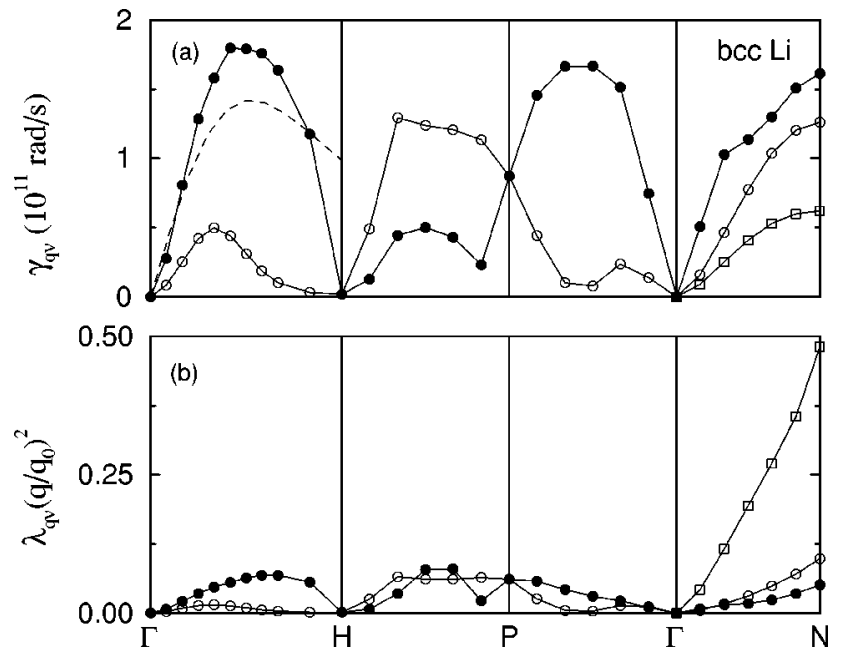

FIG. 4. (a) Phonon linewidths and (b) electron-phonon coupling parameters calculated for bcc Li along high-symmetry directions in the Brillouin zone. The coupling parameters $\lambda_{\mathbf{q} \nu}$ are weighted by a phase-space factor of $\left(q / q_{0}\right)^{2}$, where $q_{0}=2 \pi / a$. Solid circles denote longitudinal branches, while open symbols indicate transverse branches. Along the [110] direction ( $\Gamma$ to $N$ ), the open squares correspond to the $T_{1}$ branch with polarization along [1 $\left.\overline{1} 0\right]$ and the open circles correspond to the $T_{2}$ branch with polarization along [001]. The dashed curve (shown only along the [100] direction) corresponds to linewidths calculated for a free-electron model.

density of states, the bcc phase has enhanced spectral weight at low frequencies compared to the close-packed structures. Since $\lambda$ is proportional to the inverse-frequency moment of $\alpha^{2} F$, this difference at low frequencies leads to significant differences in $\lambda$. As can be seen in Table II, we find $\lambda$ $=0.45$ for bcc $\mathrm{Li}$, which is in good agreement with earlier first-principles calculations, ${ }^{21-25}$ and smaller values ranging from 0.33 to 0.37 for the three close-packed structures.

Further evidence for the importance of contributions from low-frequency modes can be found in the values of $\omega_{\log }$ tabulated in Table II. A logarithmic moment of $\alpha^{2} F$, this parameter can be expressed as $\omega_{\log }$ $=\exp \left[\Sigma_{\mathbf{q} \nu} \lambda_{\mathbf{q} \nu} \log \left(\omega_{\mathbf{q} \nu}\right) / \Sigma_{\mathbf{q} \nu} \lambda_{\mathbf{q} \nu}\right]$. Although the average phonon frequencies for the different structures differ by only about $1 \%, \omega_{\log }$ is nearly $30 \%$ smaller in bcc Li than in the close-packed phases. This indicates that some low-frequency phonon modes in bcc Li couple strongly to the electrons, thereby dramatically lowering the $\lambda$-weighted logarithmic average of the phonon frequencies.

The wave-vector- and branch-dependent phonon linewidths and coupling parameters for bcc $\mathrm{Li}$ are plotted along high-symmetry directions in Fig. 4(a). The dashed curve along the [100] direction shows the linewidths calculated for a free electron gas with the same average electron density as bcc Li. Within the simple free-electron model, which assumes a spherical Fermi surface, Thomas Fermi screening, and a single plane wave for the wave function, umklapp processes are neglected and only longitudinal phonon modes couple to electrons. The model yields a linear $q$ dependence for $\gamma$ at small wave vectors and gives a reasonable description of the calculated linewidths for longitudinal modes near the zone center. However, the full calculation finds sizable linewidths for transverse modes along all the directions plotted. 

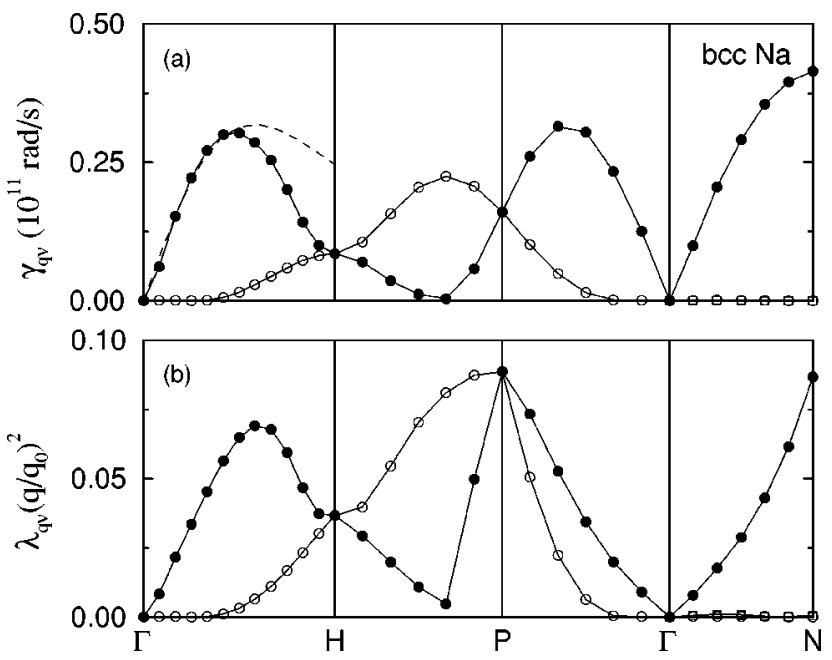

FIG. 5. (a) Phonon linewidths and (b) electron-phonon coupling parameters calculated for bcc $\mathrm{Na}$ along high-symmetry directions in the Brillouin zone. The coupling parameters $\lambda_{\mathbf{q} \nu}$ are weighted by a phase-space factor of $\left(q / q_{0}\right)^{2}$, where $q_{0}=2 \pi / a$. Solid circles denote longitudinal branches, while open symbols indicate transverse branches. Along the [110] direction ( $\Gamma$ to $N$ ), the open squares correspond to the $T_{1}$ branch with polarization along [1 $\left.1 \overline{1} 0\right]$ and the open circles correspond to the $T_{2}$ branch with polarization along [001]. The dashed curve (shown only along the [100] direction) corresponds to linewidths calculated for a free-electron model.

Figure 4(b) shows that the low-lying $T_{1}[\xi \xi 0]$ phonon modes give anomalously large contributions to $\lambda$. Yet Fig. 4(a) shows that the linewidths for these modes are comparable in magnitude to those for transverse modes along other directions. Since $\lambda_{\mathbf{q} \nu}$ is proportional to $\gamma_{\mathbf{q} \nu} / \omega_{\mathbf{q} \nu}^{2}$, and $\gamma_{\mathbf{q} \nu}$ does not depend explicitly on $\omega_{\mathbf{q} \nu}$, the large values of $\lambda_{\mathbf{q} \nu}$ associated with the $T_{1}[\xi \xi 0]$ modes arise primarily from the small phonon frequencies rather than from Fermi surface nesting or other properties related to the electronic structure.

It is interesting to compare these results for bcc $\mathrm{Li}$ to results for bcc Na, which also has a low-lying $T_{1}[\xi \xi 0]$ phonon branch. The phonon dispersion curves of bcc Na are similar to those of bcc $\mathrm{Li}$, with the frequencies scaled by the square root of the ratio of atomic masses. ${ }^{39,40}$ Yet firstprinciples calculations ${ }^{40,41}$ for bcc $\mathrm{Na}$ find $\lambda \approx 0.2$, which is less than half the value calculated for bcc Li. Since the degree of Fermi surface nesting, as measured by $\langle\langle 1\rangle\rangle$, and the electronic density of states, $N\left(E_{F}\right)$, are similar in the two systems, the differences in electron-phonon coupling arise from differences in the matrix elements. There is a particularly striking difference in the phonon linewidths along the $[\xi \xi 0]$ direction, as can be seen by comparing Figs. 4 and 5 . For both of the transverse branches along this direction, we find $\left[\gamma_{\mathbf{q} \nu} M\right]_{\mathrm{Li}} /\left[\gamma_{\mathbf{q} \nu} M\right]_{\mathrm{Na}}>1000$. This difference is due to the deeper $p$ pseudopotential in $\mathrm{Li}$ arising from the lack of $p$ states in the $\mathrm{Li}$ core. The stronger potential gives rise to a larger change in the bare potential $\delta V_{\text {bare }}$ when atoms are displaced. Furthermore, because the $p$ orbitals are more tightly bound in $\mathrm{Li}$, the electrons are less effective in screening $\delta V_{\text {bare }}$. In combination, these two effects result in significantly larger electron-phonon matrix elements in Li than in $\mathrm{Na}$. In $\mathrm{Na}$ the electrons are able to very effectively screen the change in ionic potential, particularly for the transverse

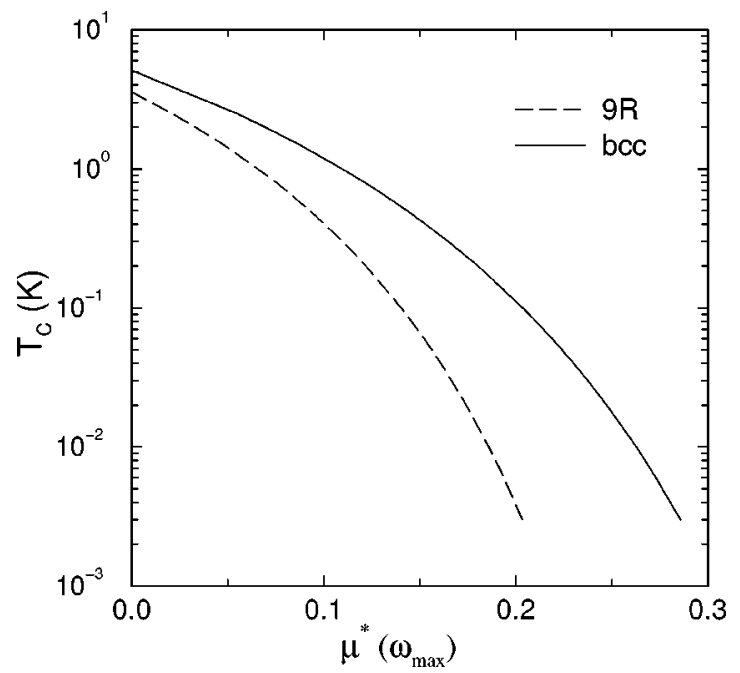

FIG. 6. Superconducting transition temperature versus $\mu^{*}$ for bcc and 9R Li. The experimental upper limit on $T_{c}$ is $6 \mathrm{mK}$.

modes along [ $\xi \xi 0]$ which contribute so strongly to $\lambda$ in $\mathrm{Li}$. Thus despite similarities in their electronic structure and phonon spectra, bcc $\mathrm{Na}$ and bcc Li differ significantly in their electron-phonon coupling parameters.

The superconducting transition temperatures calculated for bcc and $9 \mathrm{R} \mathrm{Li}$ are plotted as functions of $\mu^{*}\left(\omega_{\max }\right)$ in Fig. 6. For bcc $\mathrm{Li}$, the standard value of $\mu^{*}=0.12$ yields $T_{c}=0.8 \mathrm{~K}$, similar to earlier results in which estimates for $\lambda$ were input into the McMillan equation. As can be seen in Fig. 6, a value of $\mu^{*}=0.27$ is needed in order to suppress $T_{c}$ below the experimental limit of $6 \mathrm{mK}$. For $9 \mathrm{R} \mathrm{Li}$, the transition temperature is found to be consistent with the experimental limit if $\mu^{*}=0.19$. An approximate upper limit on $\mu^{*}$ can be obtained by letting $\mu$ become infinite in the twosquare well expression $\mu^{*}\left(\omega_{\max }\right)=\mu /\left[1+\mu \ln \left(E_{F} / \omega_{\max }\right)\right]$. For all the structures considered here, this condition leads to $\mu^{*}\left(\omega_{\max }\right)<0.23$. This confirms that the large value needed to suppress $T_{c}$ below the experimental limit in bcc $\mathrm{Li}$ is most likely unphysical. For the 9R structure, as well as the other close-packed structures, the electron-phonon interaction is weak enough to be consistent with the lack of a transition above $6 \mathrm{mK}$, provided that the Coulomb pseudopotential is somewhat larger than is commonly assumed. A realistic calculation of $\mu^{*}$ is extremely difficult since it requires knowing the full frequency- and momentum-dependent dielectric response function of the system. In a recent paper, static momentum-dependent dielectric matrices calculated within the LDA were used to estimate the Coulomb repulsion parameters in bcc and $9 \mathrm{R} \mathrm{Li}^{42}$ The estimates of $\mu^{*} \approx 0.16$ for both bcc and $9 \mathrm{R} \mathrm{Li}$ indicate that the standard values assumed for $\mu^{*}$ are not appropriate for this material. This is also supported by a recent study of solutions to the Eliashberg equation for the electron gas which found that the role of Coulomb repulsion is significantly underestimated in lowdensity metals if $\mu^{*} \approx 0.1$ is used. ${ }^{43}$

The thermal effective mass derived from heat capacity measurements provides additional information on the electron-phonon mass enhancement parameter. The thermal mass is related to the band mass according to $m_{\mathrm{th}}=m_{b}(1$ $+\lambda)$ and is found experimentally from the electronic contribution to the specific heat. Measurements for Li yield $m_{\text {th }}$ 
$=2.22 m$, where $m$ is the bare electron mass. ${ }^{20}$ The band mass for $9 \mathrm{R} \mathrm{Li}$ is calculated to be about $7 \%$ larger than that for bcc Li, which offsets the difference in $\lambda$. The calculated thermal masses of $m_{\mathrm{th}}=2.25 \mathrm{~m}$ and $2.24 m$ for bcc and $9 \mathrm{R} \mathrm{Li}$, respectively, are both in good agreement with the specific heat data. Note however that this analysis does not place strict constraints on $\lambda$ since contributions to the mass renormalization arising from electron-electron manybody effects beyond the LDA have been assumed to be negligible.

Our results for the electron-phonon interaction in $9 \mathrm{R} \mathrm{Li}$ are thus consistent both with the observed lack of a superconducting transition as well as with the specific heat data. One complication is that since $\mathrm{Li}$ is never observed to transform completely to a perfect $9 \mathrm{R}$ lattice-there is always a mixture of the disordered close-packed polytype, as well as some amount of bcc, the exact amounts of which depend on thermal history - the exact structural characteristics of the samples used in the various experiments are not known. Depending on the details of how grains with different crystal structures are distributed within the samples, the structural disorder itself may play a role in the suppression of superconductivity in this material. We hope this work motivates future experimental studies on well-characterized samples of low-temperature $\mathrm{Li}$ and on possible connections between the microstructure of the samples and electronic and transport properties.

\section{CONCLUSIONS}

We have used the first-principles linear-response method to compute the vibrational properties of $\mathrm{Li}$ in various crystal structures. The low-energy $T_{1}[\xi \xi 0]$ phonon modes, which correspond to shearing of (110) planes, distinguish the vibrational properties of the bcc phase from those of the closepacked phases. In particular, while the close-packed 9R, fcc, and hcp phases are energetically favorable at $T=0$, the large phonon entropy associated with the low-energy modes in bcc $\mathrm{Li}$ stabilizes the bcc structure upon heating. The quasiharmonic approximation gives a reasonable description of the thermodynamics of the transformation at zero pressure, yielding a transition temperature within about $100 \mathrm{~K}$ of the observed starting temperature $M_{s}$.

The thermodynamic properties addressed in this work provide a starting point for understanding the details of the structural transformation in Li. Many interesting questions remain about the kinetics of the transition, including for example, details about the reaction path, ${ }^{44}$ the role played by coherency stresses in determining which close-packed phase appears, ${ }^{4}$ the appearance of the fcc phase intermediate between 9R and bcc upon heating, ${ }^{4}$ and the importance of heterophase fluctuations and defects for triggering the transition. ${ }^{45,46}$

The $T_{1}[\xi \xi 0]$ phonon modes which are responsible for stabilizing the bcc phase at high temperatures also play an important role in the variation of the electron-phonon coupling strength with crystal structure. It is primarily contributions from these modes that make the coupling parameter $\lambda$ about $30 \%$ larger in bcc Li than in the close-packed phases. The coupling in the 9R structure, which is the predominant phase at low temperatures, is weak enough to be consistent with the current experimental upper limit on $T_{c}$, provided that a value of $\mu^{*}\left(\omega_{\max }\right) \approx 0.19$ is assumed. This is a plausible value given recent theoretical work that shows that the Coulomb repulsion parameter in low-density metals like Li can significantly exceed the standard values assumed. ${ }^{42,43}$ The calculated enhancement of the electronic mass due to the electron-phonon interaction in $9 \mathrm{R} \mathrm{Li}$ is also consistent with available specific heat data.

\section{ACKNOWLEDGMENTS}

We thank D.W. Hess and B. Sadigh for helpful discussions. This work was supported by National Science Foundation Grant No. DMR9627778 and by the United States Department of Energy, Office of Basic Science, Division of Materials Science. A.Y.L. acknowledges the support of the Clare Boothe Luce Fund. E.J.N. was supported by the Research Corporation and by the Natural Sciences and Engineering Research Council of Canada. Supercomputing time was provided by the NSF at the Pittsburgh Supercomputing Center and the San Diego Supercomputer Center.
${ }^{1}$ C. S. Barrett, Acta Crystallogr. 9, 671 (1956).

${ }^{2}$ A. W. Overhauser, Phys. Rev. Lett. 53, 64 (1984).

${ }^{3}$ H. G. Smith, Phys. Rev. Lett. 58, 1228 (1987).

${ }^{4}$ W. Schwarz and O. Blaschko, Phys. Rev. Lett. 65, 3144 (1990).

${ }^{5}$ V. G. Vaks, M. I. Katsnelson, V. G. Koreshkov, A. I. Likhtenstein, O. E. Parfenov, V. F. Skok, V. A. Sukhoparov, A. V. Trefilov, and A. A. Chernyshov, J. Phys.: Condens. Matter 1, 5319 (1989).

${ }^{6}$ H. G. Smith, R. Berliner, J. D. Jorgensen, M. Nielsen, and J. Trivisonno, Phys. Rev. B 41, 1231 (1990).

${ }^{7}$ B. Olinger and J. W. Shaner, Science 219, 1071 (1983).

${ }^{8}$ G. Ernst, C. Artner, O. Blaschko, and G. Krexner, Phys. Rev. B 33, 6465 (1986).

${ }^{9}$ H. G. Smith, R. Berliner, and J. Trivisonno, Phys. Rev. B 49, 8547 (1994).

${ }^{10}$ C. Zener, Phys. Rev. 71, 846 (1947).

${ }^{11}$ W. Cochran, Adv. Phys. 9, 387 (1960).
${ }^{12}$ P. W. Anderson, in Fizika Dielectrikov, edited by G. I. Skanavi (Akad. Nauk SSR, Moscow, 1960).

${ }^{13}$ J. Friedel, J. Phys. (France) Lett. 35, L59 (1974).

${ }^{14}$ J. C. Boettger and S. B. Trickey, Phys. Rev. B 32, 3391 (1985); M. M. Dacorogna and M. L. Cohen, ibid. 34, 5065 (1986); H. Bross and R. Stryczek, Phys. Status Solidi B 144, 675 (1987); J. C. Boettger and R. C. Albers, Phys. Rev. B 39, 3010 (1989); J. A. Nobel, S. B. Trickey, P. Blaha, and K. Schwarz, ibid. 45, 5012 (1992).

${ }^{15}$ P. Staikov, A. Kara, and T. S. Rahman, J. Phys.: Condens. Matter 9, 2135 (1997).

${ }^{16}$ A. A. Maradudin, E. W. Montroll, G. H. Weiss, and I. P. Ipatova, Theory of Lattice Dynamics in the Harmonic Approximation, 2nd ed. (Academic, New York, 1971).

${ }^{17}$ A. A. Quong and A. Y. Liu, Phys. Rev. B 56, 7767 (1997).

${ }^{18}$ S. Baroni, P. Giannozzi, and A. Testa, Phys. Rev. Lett. 58, 1861 (1987). 
${ }^{19}$ A. A. Quong and B. M. Klein, Phys. Rev. B 46, 10734 (1992).

${ }^{20}$ N. E. Phillips, Crit. Rev. Solid State Sci. 2, 467 (1971).

${ }^{21}$ D. A. Papaconstantopoulos, L. L. Boyer, B. M. Klein, A. R. Williams, V. L. Morruzzi, and J. F. Janak, Phys. Rev. B 15, 4221 (1977).

${ }^{22}$ T. Jarlborg, Phys. Scr. 37, 795 (1988).

${ }^{23}$ G. Grimvall, The Electron-Phonon Interaction in Metals (NorthHolland, Amsterdam, 1981).

${ }^{24}$ A. Y. Liu and M. L. Cohen, Phys. Rev. B 44, 9678 (1991).

${ }^{25}$ A. Y. Liu and A. A. Quong, Phys. Rev. B 53, R7575 (1996).

${ }^{26}$ W. L. McMillan, Phys. Rev. 167, 331 (1968).

${ }^{27}$ T. L. Thorp, B. B. Triplett, W. D. Brewer, M. L. Cohen, N. E. Phillips, D. A. Shirley, J. E. Templeton, R. W. Stark, and P. H. Schmidt, J. Low Temp. Phys. 3, 589 (1970); K. M. Lang, A. Mizel, J. Mortara, E. Hudson, J. Hone, M. L. Cohen, A. Zettl, and J. C. Davis, ibid. (to be published).

${ }^{28}$ N. Troullier and J. L. Martins, Phys. Rev. B 43, 1993 (1991).

${ }^{29}$ E. Wigner, Phys. Rev. 46, 1002 (1934).

${ }^{30}$ S. G. Louie, S. Froyen, and M. L. Cohen, Phys. Rev. B 26, 1738 (1982).

${ }^{31}$ N. D. Mermin, Phys. Rev. 137, A1441 (1965).

${ }^{32}$ F. Birch, J. Geophys. Res. 83, 1257 (1978).

${ }^{33}$ Upon heating, the low-temperature mixture of $9 \mathrm{R}$ and other close- packed phases appears to first transform to fcc around $100 \mathrm{~K}$, and then to bcc above about $150 \mathrm{~K}$ (Ref. 4).

${ }^{34}$ M. J. Mehl, Phys. Rev. B 47, 2493 (1993).

${ }^{35}$ Y.-Y. Ye, Y. Chen, K.-M. Ho, B. N. Harmon, and P.-A. Lindgard, Phys. Rev. Lett. 58, 1769 (1987).

${ }^{36}$ P. K. Lam, M. M. Dacorogna, and M. L. Cohen, Phys. Rev. B 34, 5065 (1986).

${ }^{37}$ Y. S. Touloukian et al., Thermophysical Properties of Matter (Plenum, New York, 1979), Vol. 12.

${ }^{38}$ J. P. Carbotte, Rev. Mod. Phys. 62, 1027 (1990).

${ }^{39}$ W. Frank, C. Elsasser, and M. Fahnle, Phys. Rev. Lett. 74, 1791 (1995).

${ }^{40}$ A. Y. Liu and A. A. Quong (unpublished).

${ }^{41}$ R. Bauer, A. Schmid, P. Pavone, and D. Strauch, Phys. Rev. B 57, 11276 (1998).

${ }^{42}$ Y. G. Jin and K. J. Chang, Phys. Rev. B 57, 14684 (1998).

${ }^{43}$ C. F. Richardson and N. W. Ashcroft, Phys. Rev. B 54, R764 (1996).

${ }^{44}$ Y.-Y. Ye, C. T. Chan, K.-M. Ho, and B. N. Harmon, Int. J. Supercomput. Appl. 4, 111 (1990).

${ }^{45}$ D. A. Vul and B. N. Harmon, Phys. Rev. B 48, 6880 (1993).

${ }^{46}$ J. R. Morris and R. J. Gooding, Phys. Rev. B 43, 6057 (1991). 